\title{
Value of Orthostatic Hypotension as a Prognostic Bed-Side Test in Heart Failure*
}

\author{
Tarek M. Abdel Rahman \\ Cardiology Department, El Minia University Hospital, Minya, Egypt \\ Email: tarekabdelrahman2@yahoo.com
}

Received July 27, 2012; revised August 28, 2012; accepted September 6, 2012

\begin{abstract}
Background: Neurohumoral compensatory mechanisms play an important role in stabilizing the functional activity of patients with heart failure using the arms of autonomic nervous system. Orthostatic Hypotension $(\mathrm{OH})$ is one of the most incapacitating symptoms of Cardiac Autonomic Dysfunction (CAD). OH can include sympathetic withdrawal which in turn leads to marked disability and deterioration of heart failure symptoms. Progressive Autonomic Dysfunction (AD) associated with progressive deterioration and impact on mortality of many diseases as hypertension, diabetes and other chronic diseases. The idea of using $(\mathrm{OH})$ as a bed-side simple test expecting the risk of deterioration of cardiac function and furthermore on mortality open a gateway for preventive medicine and care to these group of patients. For more confidential prove, studying subjective and objective factors in heart failure patients became necessary to support these idea. Methods and Results: Sixty-Four patients with known history of heart failure were collected. All patients taking the fixed regiment of 4 drugs (diuretic, ACE inhibitor, Digitalis and B-blocker) in appropriate tolerated doses for two weeks prior to the study. History taking and all routine investigations were done for all patients. Grouping is based upon wither they have $(\mathrm{OH})$ or not. Group-A found to have normal BP response to standing; they were 24 patients (18 male and 6 female) of mean Age ( $45 \pm 8$ years). Group-B discovered to have significant $(\mathrm{OH})$ and was 22 patients $(16$ males and 6 females) of mean Age (43 \pm 4 years). The first Clinical and Echocardiographic examination was done and considered as a base-line characteristic. Then, a Call-back after 6 months for follow-up and second visit examination is recorded. Furthermore, every patient was advised to report changes in his clinical symptoms in a note-book describing five main items to answer a questionnaire at the end of the study involves [times of admission to hospital, need for treatment modification, numbers of paroxysmal nocturnal dysnea, numbers of arrhythmic episodes and manifest lower limb edema]. At the end of the study, statistical methods are used to clarify the changes in their data and detect deterioration of cardiac functions by Echocardiographic results and their answers to the questionnaire. In the first visit, comparison of demographic, clinical and Echocardiographic data revealed no significant variations - odd values are excluded and the remaining 46 patients are then tested for their Bp response to standing and classified into two groups. Group-A (normal Bp response) and Group-B (having orthostatic hypotension). Group-A were 24 patients (18 male and 6 female) of mean Age ( $45 \pm 8$ years). Group-B were 22 patients (16 males and 6 females) of mean Age (43 \pm 4 years). In the second visit (6-month later), divergence of data is observed and was statistically significant. Group-B was found to have a lower EF\% and FS\% $(p=0.01)$, a lower Dp/Dt $(p=0.01)$ and a higher Tie-Index and MR-jet area $(p=0.01)$. This means that, patients having orthostatic hypotension underwent significant deterioration of cardiac functions after a period of six-months. Indeed, the questionnaire proved frequent times of hospital admissions, paroxysmal nocturnal dysnea, need for treatment modification, arrhythmias and lower limb edema in group-B. Discussion: The present study conclude that, heart failure-patients having orthostatic hypotension experienced a significant deterioration of clinical condition and cardiac functions along a period of six-months which represent failure in their autonomic compensatory mechanisms and possible impact on their mortality. However, extended trials on mortality are needed to confirm this idea. Otherwise, we might use $(\mathrm{OH})$ as a simple, rapid, bed-side tool to determine their prognosis and directing the physician attention to close care towards these subset of patients.
\end{abstract}

Keywords: Orthostatic; Hypotension; Heart Failure

"Funding sources: All the study fees were sponsored by the governmental policies to support the research work in my university according to the established financial protocols; Ethics Approval: All patients wrote a written approval to be included in the research work. 


\section{Introduction}

The Central blood volume is abruptly reduced when one stands up. A complex set of compensatory physiological mechanisms then occur to maintain the upright posture. These include reflex responses in the cardiovascular and autonomic nervous systems as well as activation of the skeletal muscle and respiratory pumps. As a result, rapid changes in arterial blood pressure occur [1]. In population studies, the Systolic Blood Pressure (SBP) response to a change in posture is approximately normally distributed with a mean close to $0 \mathrm{~mm} \mathrm{Hg}$, but the range includes SBP decreases and increases of considerable magnitude [2].

Orthostatic Hypotension $(\mathrm{OH})$ occurs when there is a marked decrease in blood pressure after the upright posture is assumed. Although previously not consistently defined, guidelines established in the 1990s suggested defining $\mathrm{OH}$ as a decrease in SBP $\geq 20 \mathrm{~mm} \mathrm{Hg}$ and/or a decrease in Diastolic Blood Pressure (DBP) $\geq 10 \mathrm{~mm} \mathrm{Hg}$ [3].

In middle-aged persons in the Atherosclerosis Risk in Communities (ARIC) Study, $\mathrm{OH}$ has been associated with incident hypertension, Coronary Heart Disease (CHD) [4], and stroke [5]. Several studies have examined the association between $\mathrm{OH}$ and mortality in the elderly or other high-risk populations. Some have reported a modest increased risk of mortality among those with $\mathrm{OH}$ [6-8], whereas others have reported no association [9].

Orthostatic Blood Pressure (BP) control involves complex compensatory mechanisms allowing the human body to stand upright [10]. As the postural homeostasis is principally mediated by autonomic nervous system, its impairment may lead to BP fall after standing. The phenomenon, denominated as Orthostatic Hypotension $(\mathrm{OH})$, is often associated with debilitating symptoms: fatigue, dizziness, and fainting [11]. Orthostatic hypotension has been defined by the international consensus as a decrease in systolic BP $\geq 20 \mathrm{mmHg}$ and/or decrease in diastolic BP $\geq 10 \mathrm{mmHg}$ within $3 \mathrm{~min}$ of standing [3]. In addition, some authors have proposed standing systolic BP $<90$ $\mathrm{mmHg}$ as an alternative criterion [12].

Clinicians are usually interested in diagnosing $\mathrm{OH}$ as it can cause fall-related injuries, substantially limit patients' quality of life [13], and finally, impede relevant treatment of concomitant diseases as hypertension or heart failure [14]. In parallel, relatively little is known about prognostic aspects of $\mathrm{OH}$. Increased mortality and incidence of cardiovascular disease (CVD) related to prevalent $\mathrm{OH}$ has been reported in different high-risk groups with dominantly symptomatic patients. However, the number of studies regarding the prognostic value of younger and mainly asymptomatic individuals without significant burden of co-morbidities is limited. Prospective data from the Atherosclerosis Risk in Communities (ARIC) study suggest that $\mathrm{OH}$ may confer higher risk of all-cause mortality and cardiovascular events [15-18].
Cardiovascular Autonomic Neuropathy (CAN) is progressive and starts without symptoms. The pre-symptomatic state is defined as "Autonomic Dysfunction (AD)".

As AD increases in severity, it leads to peripheral autonomic neuropathy, then Diabetic Autonomic Neuropathy (DAN), and, finally, symptoms associated with end-organ failure. This final stage is also known as CAN [19].

In patients with diabetes, autonomic assessment is often based on three time-domain Heart Rate Variability (HRV) ratios: the Exhalation/Inhalation (E/I) ratio from a deep breathing challenge, the Valsalva ratio, and the 30:15 ratio from an upright posture challenge [33]. DAN is indicated if two of the three ratios are abnormally low. CAN is indicated if all three ratios are low. DAN is a risk factor for CAN, and both DAN and CAN are associated with Blood Pressure (BP) anomalies, including Orthostatic Hypotension $(\mathrm{OH})[20,21]$.

Orthostatic hypotension is one of the most incapacitating symptoms of autonomic failure, including CAN [22], and may be used as a marker for CAN. In diabetes and other chronic diseases, AD and CAN are also associated with orthostatic symptoms. Orthostasis presents earlier in patients with diabetes than in those without [23]. The earlier onset of orthostasis is partly due to the fact that diabetes can accelerate $\mathrm{AD}$ and therefore $\mathrm{CAN}$ onset. There is a wide variability in the diagnosis of orthostasis, especially in earlier stages when therapy can be lower dose and shorter term [24].

Orthostasis may be difficult to detect and diagnose because both Autonomic Nervous System (ANS) branches are actively changing in a coordinated fashion during a normal response to postural change: the parasympathetics decrease or withdrawal and the sympathetics increase or surge [22,24,25]. Therefore, diagnosing orthostasis requires a test that independently and simultaneously measures the response of both ANS branches, the sympathetic nervous system and the parasympathetic nervous system. Because CAN is treatable in all stages and because orthostasis is often treatable in parallel with the other effects of AD [26], the American Diabetes Association and the American Heart Association have recommended testing diabetic patients more than once per year to detect symptoms as early as possible to slow progression of $\mathrm{AD}[27,28]$.

\section{Materials and Methods}

The study was carried out in the Cardiology Dep. of ElMinia University hospital from the period of 1st January to $1^{\text {st }}$ September 2011. Patients were recruited from outpatient clinic and archived as regards their names, tel. number, address, and results of their clinical examination to be stored as a base-line data, then advised to follow-up after 6 months. Data were obtained and computed for easy comparing the data of their examination. 


\subsection{The Study Population Is Designed as Follows}

Selection criteria, were to choose Patients known as or first discovered to have symptoms and signs of cardiac decompensation and diagnosed as idiopathic or Ischaemic heart failure based on Echocardiography. A 46 patients were collected with mean age ( $44 \pm 6$ years old). All patients taking the fixed regiment of 4 drugs (diuretic, ACE inhibitor, Digitalis and B-blocker) in appropriate tolerated doses for 15 days and then the first examination was done and considered as a base-line characteristics. Then, a Call-back after 6 months for follow-up and second visit examination is recorded.

Exclusion criteria, were valvular, congenital, pericardial and hypertensive heart failure. Frail patients or poor health individuals are excluded. Co morbidities (CHD, stroke, cancer, Epileptic seizures, hypertension, diabetes, or a self-reported fair/poor health status) are also excluded from the study population. None of them taking specific drugs such as (centrally acting drugs, tri-cyclic antidepressant, anti-hypertensive or hypoglycemic agents, cold remedies or thyroid related agents). None of them is current smoker.

\subsection{The Methodology Is Designed as Follows}

All patients were examined as full-history taking, ECG, general and cardiac examination. Echocardiography was done and the following data were obtained (LA and LV dimensions, $\mathrm{EF} \%$ and $\mathrm{FS} \%$, Dp/Dt in msec, Tei index and MR jet area).

LA Dimension: internal chamber dimensions are taken from parasternal long axis view from the leading the leading edge to trailing edge as shown in Figure 1.

Lv Dimensions, $\mathrm{FS} \%$ and EF\%: are taken from MMode in Parasternal long axis view from the trailing edge to leading edge as shown in Figure 2.

$\mathrm{Dp} / \mathrm{Dt}$ : is the rate of pressure rise inside left ventricle and is taken from the MR in continuous Doppler mode of the sample volume adjusted in the center of MV orifice as shown in Figure 3.

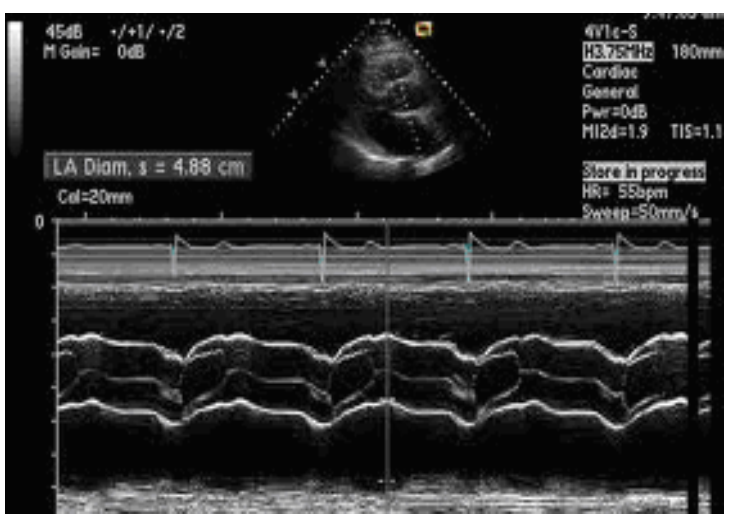

Figure 1. LA dimension from parasternal lax view.
Tei-Index: is the Index of myocardial performance which is devised to incorporate both systolic and diastolic time intervals in expressing global ventricular performance. systolic dysfunction results in a prolongation of the preejection time (IVCT) and a shortening of the Ejection Time (ET). Both systolic and diastolic dysfunction result in abnormality in myocardial relaxation, which prolongs the relaxation period (IVRT). So, Tei-Index $=[$ IVCT + IVRT]/ET. Normal value is $0.39 \pm 0.05$ as shown in Figure 4.

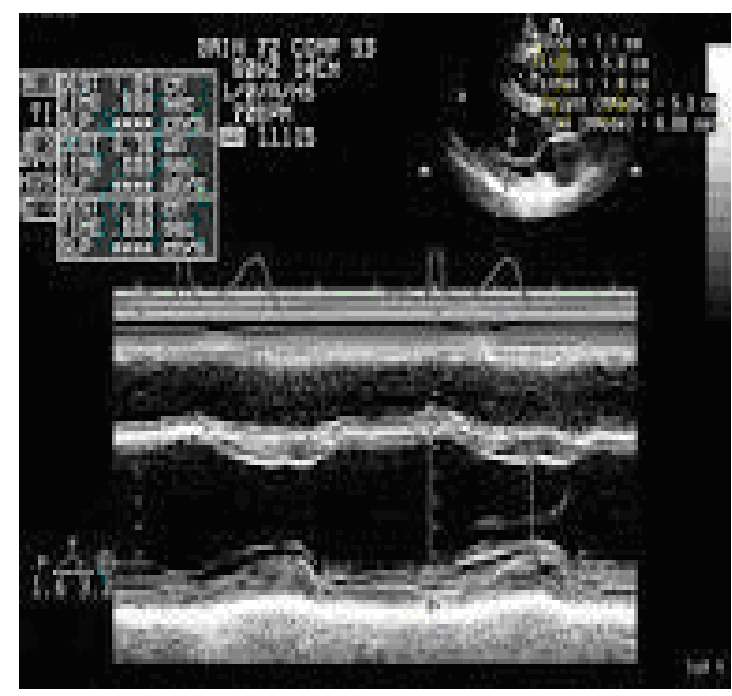

Figure 2. Lv Dimensions from parasternal lax view.

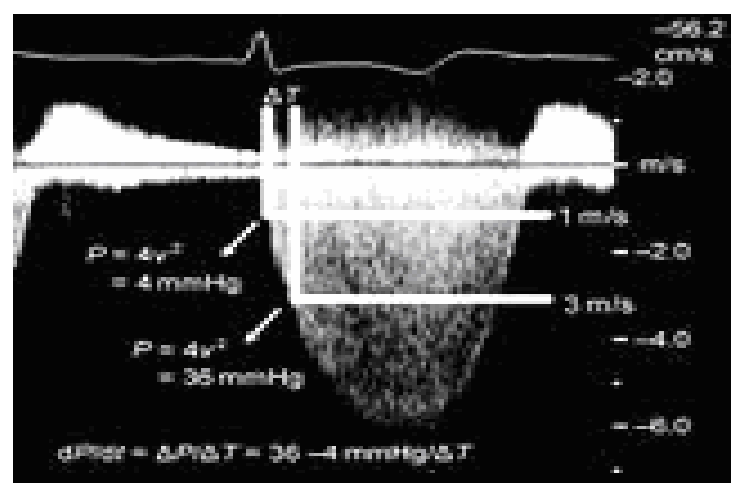

Figure 3. Dp/Dt from doppler mode of mitral flow.

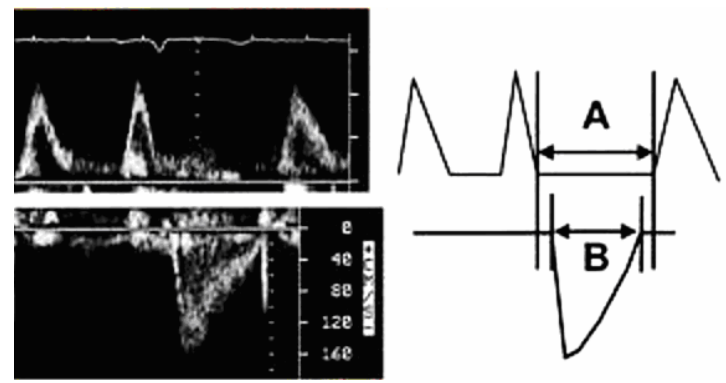

Figure 4. Tei-Index: A/B however, $A=(I V C T+I V R T)$ and $B=($ ejection time). 
MR jet area: is measured in tracing the color-jet of MR in systole by two methods (parasternal and 2D) after adjusting the aliasing and Nyquist limit in colored Mode and averaged as shown in Figure 5.

Data were averaged in patients with atrial fibrillation rythm. After a period of six months, all data of the patients were compared for each patient in relation to his individual base-line data. In addition each patient advised to report changes in his clinical symptoms in a note-book describing five main items to answer a questionnaire at the end of the study involves (times of admission to hospital, need for treatment modification, numbers of paroxysmal nocturnal dysnea, numbers of arrhythmic episodes and manifest lower limb edema).

\subsection{Measurement and Classification of Blood Pressure Response to a Change in Posture}

We used two methods to measure supine and standing blood pressure.

First, was Automated blood pressure measurements whish was taken approximately every 30 seconds for 2 minutes (range of 2 to 5 measurements; $90 \%$ had at least 4 measurements). Participants were then asked to stand, and as their feet touched the ground, a standing blood pressure measurement was taken. Measurements were repeated during the first 2 minutes after standing (range of 2 to 5 measurements).

Because blood pressure restabilization is still occurring during the first 30 seconds after standing [29], blood pressure change was defined as the difference between the average of the standing and the supine blood pressure measurements, excluding the first standing measurement. With the use of established guidelines [3], participants were classified by the presence (a decrease of at least 20 $\mathrm{mmHg}$ SBP or a decrease of at least $10 \mathrm{mmHg}$ DBP) or absence of $\mathrm{OH}$.

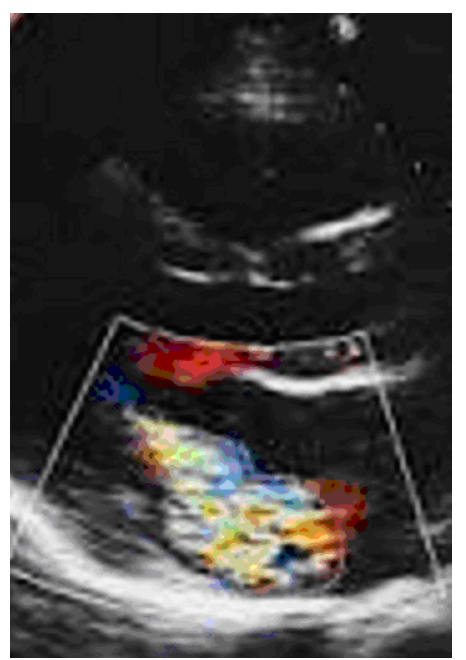

Figure 5. MR jet area tracing from Parasternal LAx view.
Second reading was obtained in another setting in the same hour, after doing the Echocardiography in which the Blood pressure ( $\mathrm{mmHg}$ ) was re-measured auscultatory by specially trained nurses in two different positions (supine and standing), with a mercury sphygmomanometer and an appropriate cuff placed around the right arm. First BP reading was taken after $10 \mathrm{~min}$ rest in the supine position. Then the participants were asked to stand up and the second BP measurement was taken after 1 min. Blood pressure was determined and recorded to the nearest $5 \mathrm{mmHg}$. The final record was through approximation of each figure to another, but, if marked difference $>10 \mathrm{mmhg}$, the auscultatory method is chosen.

\subsection{Definition of Orthostatic Hypotension}

Orthostatic hypotension at the baseline was defined according to the international consensus [3] as a decrease in systolic BP $\geq 20 \mathrm{mmHg}$ and/or decrease in diastolic BP $\geq 10 \mathrm{mmHg}$ within $3 \mathrm{~min}$ of standing, or, in addition, as standing systolic BP $<90 \mathrm{mmHg}$.

\subsection{The Study Grouping}

Patient's Data were collected and results of blood pressure response to standing classify the patients into two groups; individuals with a normal response to standing (OH-negative) were defined as Group-A and individuals with orthostatic hypotension ( $\mathrm{OH}$-positive) were defined as Group-B. The group-A were 24 patients of mean Age $(45 \pm 8$ y) and group-B were 22 patient of mean Age (43 $\pm 4 \mathrm{y})$.

Clinical and Echocardiographic data were compared in relation to base-line and 6-months follow up results. Moreover, questionnaire is applied and their answers were also compared.

\section{Results}

Clinical Data were compared as regards the base-line between group-A and group-B and revealed no significant variations as shown in Table 1.

Table 1. Demographic and clinical data of both groups (A, B).

\begin{tabular}{|c|c|c|c|c|c|}
\hline \multirow{2}{*}{ Parameter } & \multicolumn{2}{|c|}{ Group-A } & \multicolumn{2}{|c|}{ Group-B } & \multirow{2}{*}{ P-value } \\
\hline & Mean & $\mathrm{SD}$ & Mean & $\mathrm{SD}$ & \\
\hline Age & 45 & 8 & 43 & 4 & NS \\
\hline $\begin{array}{l}\text { Sex } \\
\quad \text { - Males } \\
\text { - Females }\end{array}$ & & & & & NS \\
\hline BMI & 28.2 & 2.6 & 26.8 & 4.8 & NS \\
\hline NYHA-Class & 2.6 & 0.64 & 2.8 & 0.44 & NS \\
\hline Systolic Bp & 118.1 & 14.8 & 116.2 & 15.2 & NS \\
\hline Heart Rate & 95.2 & 10.0 & 96.2 & 8.8 & NS \\
\hline
\end{tabular}


Also, Echocardiographic Data were compared in the first visit between group-A and group-B and revealed no significant variations as shown in Table 2.

Echocardiographic Data were compared between baseline features and 6-months follow-up visit and revealed a significant deterioration in group-B in the form of reduction in $\mathrm{EF} \%$ \& $\mathrm{FS} \%$, reduced $\mathrm{Dp} / \mathrm{Dt}$, prolongation of TeiIndex and increased MR-jet area in group-B in relation to group-I as shown in Table 3.

Graphic presentations of the Echocardiographic results display this deterioration at the end of the study as shown in the following Figures 6-8.

Table 2. Echocardiographic data at first-visit base line in both groups.

\begin{tabular}{lccccc}
\hline \multirow{2}{*}{ Parameter } & \multicolumn{2}{c}{ Group-A } & \multicolumn{2}{c}{ Group-B } & \multirow{2}{*}{ P-value } \\
\cline { 2 - 5 } & Mean & SD & Mean & SD & \\
\hline EF \% & 40.2 & 2.5 & 39.5 & 1.9 & NS \\
FS \% & 20.4 & 1.4 & 19.8 & 1.3 & NS \\
Dp/Dt & 742.3 & 22.6 & 652.3 & 10.7 & NS \\
Tei-Index & 0.54 & 0.18 & 0.62 & 0.08 & NS \\
MR-jet area & 3.32 & 0.16 & 3.4 & 0.12 & NS \\
\hline
\end{tabular}

Table 3. Echocardiographic data between groups after 6 months.

\begin{tabular}{lccccc}
\hline \multirow{2}{*}{ Parameter } & \multicolumn{2}{c}{ Group-A } & \multicolumn{2}{c}{ Group-B } & \multirow{2}{*}{ P-value } \\
\cline { 2 - 5 } & Mean & SD & Mean & SD & \\
\hline EF \% & 41.0 & 2.2 & 38.2 & 1.6 & 0.01 \\
FS \% & 20.8 & 1.6 & 18.4 & 1.1 & 0.01 \\
Dp/Dt & 762.1 & 23.5 & 644.3 & 40.2 & 0.001 \\
Tei-Index & 0.48 & 0.13 & 0.71 & 0.18 & 0.001 \\
MR-jet area & 3.55 & 0.14 & 4.2 & 0.21 & 0.01 \\
\hline
\end{tabular}

Comparison of EF and FS in both Groups

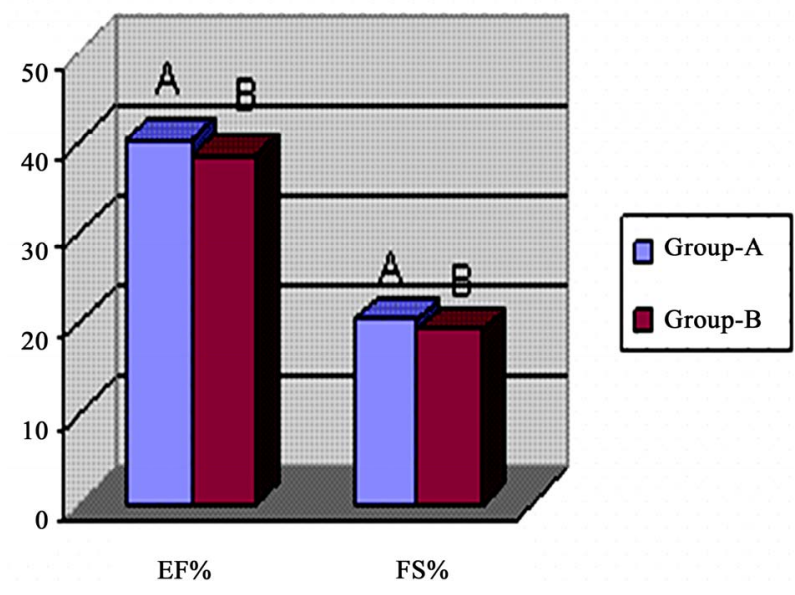

Figure 6. Revealed higher EF\% and FS\% in group-A than group-B.
Moreover, answers of the questionnaire at the end of the study revealed that, Group-B who was positively having orthostatic hypotension experienced frequent admission to hospital, frequent attacks of PND and palpitations. Also, observed increased times of manifest lower limb edema and need many times to increase their diuretic doses as shown in Table 4.

\section{Discussion}

Cardiovascular Autonomic Neuropathy (CAN) is progressive disease and starts without symptoms. The presymptomatic state is defined as "Autonomic Dysfunction (AD)". Orthostatic hypotension is one of the most incapacitating symptoms of autonomic failure, including CAN [30], and may be used as a marker for CAN. In diabetes and other chronic diseases, $\mathrm{AD}$ and $\mathrm{CAN}$ are also associated with orthostatic symptoms [31].

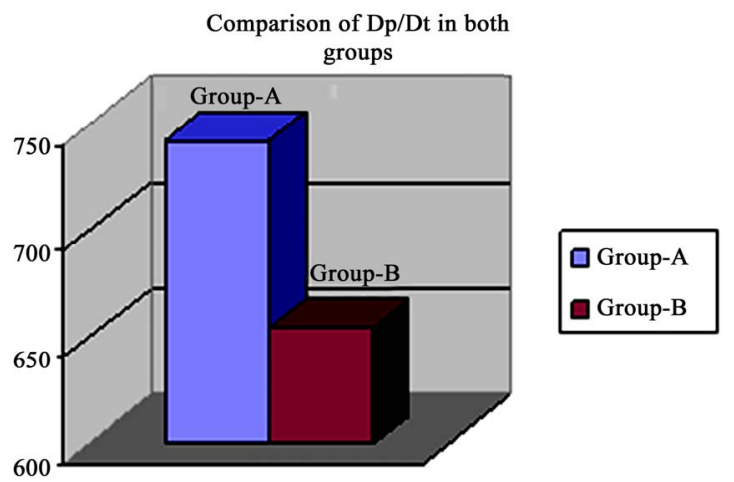

Figure 7. Revealed higher Dp/Dt in group-A than group-B.

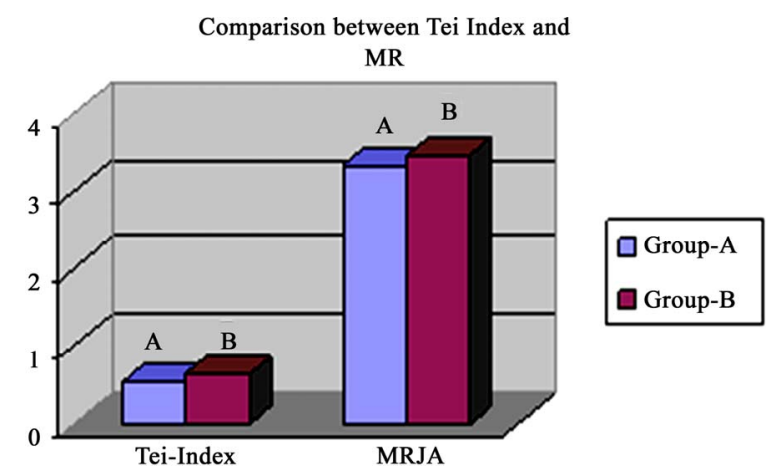

Figure 8. Revealed higher Tei Index and MR jet area in group-B than group-A.

Table 4. Answers of the questionnaire.

\begin{tabular}{lccc}
\hline \multicolumn{1}{c}{ Question } & Group A & Group B P value \\
\hline Numbers of Admission to hospital? & 2 & 6 & 0.001 \\
Attacks of Paroxysmal Nocturnal Dysnea? & 0 & 3 & 0.001 \\
Need for Treatment Modifications? & 1 & 4 & 0.001 \\
Attacks of Arrhythmias or Palpitations? & 1 & 4 & 0.001 \\
Manifest lower limb edema? & 1 & 6 & 0.001 \\
\hline
\end{tabular}


There is a wide variability in the diagnosis of orthostasis, Orthostasis may be difficult to detect and diagnose because both Autonomic Nervous System (ANS) branches are actively changing in a coordinated fashion during a normal response to postural change: the parasympathetics decrease or withdrawal and the sympathetics increase or surge. Therefore, diagnosing orthostasis requires a test that independently and simultaneously measures the response of both ANS branches, the sympathetic nervous system and the parasympathetic nervous system [31].

Most research on $\mathrm{OH}$ is based on elderly, frail populations in which $\mathrm{OH}$ is accompanied by symptoms of dizziness and syncope and is associated with falls, fractures, and potential serious morbidity. Recent evidence suggests that $\mathrm{OH}$ in the elderly is associated with decreases in vestibular function. $\mathrm{OH}$ is consistently associated with older age, elevated blood pressure, and thicker carotid arterial walls. Inconsistent associations are noted between $\mathrm{OH}$ and Body Mass Index (BMI), diabetes, and cigarette smoking. Few prospective studies have investigated the association between $\mathrm{OH}$ and cardiovascular disease outcomes [32,33].

Research studies should be directed towards patients with known structural heart pathology after excluding other non-specific factors and its implication on prognosis and outcome of these cardiac diseases, our study aiming to put idea on this value choosing heart failure as a variant of cardiac patients having much more incapacitations and short life span.

Our results were promising through identifying the group of heart failure patients experienced $(\mathrm{OH})$ in their blood pressure recordings and confirmed a much more deterioration in their clinic or Echocardiographic features. The group-A showed significant higher $\mathrm{EF} \%, \mathrm{FS} \%$ and $\mathrm{Dp} / \mathrm{Dt}$ than Group-B after six months, in spite of, being nearly similar in the first visit and this finding was identical to the results of O. Almoznino et al., in 2009 who reports the same findings and recommends the idea of using Leg compression bandaging thinking of that is may be useful for prevention of postural hypotension in these patients [34].

Recently, in a Swedish prospective cohort study done over a period of 24 years by Fedorowski et al., in 2011, discovered the idea of effect of $(\mathrm{OH})$ in determining the course and outcome of heart failure deterioration. The study concluded that, the incidence of first hospitalization and new-onset HF was related to early postural changes in systolic and diastolic blood pressure and prove that early increase of blood pressure in response to orthostatic challenge signals reduced the risk of HF development.[35]

Before one year the same author detect the link between $\mathrm{OH}$ and all cause mortality as, Orthostatic hypotension can be detected in $6 \%$ of middle-aged individuals and is often associated with such comorbidities as hypertension or diabetes. Presence of $\mathrm{OH}$ increases mortality and $\mathrm{CE}$ risk, independently of traditional risk factors. Although both impaired systolic and diastolic responses predict adverse events, the diastolic impairment shows stronger association with coronary disease [36].

Also, Potocka et al., in 2001 confirmed that, CHF patients showed a decreased ability to develop compensatory tachycardia during hypotension. Moreover, reduction in systolic blood pressure was more pronounced in CHF patients, and diastolic blood pressure increase was less significant as compared with his control group [37].

Our study revealed more clinical deterioration in group-B in the form of frequent hospitalizations and need for intensifying medical treatment with recurrent attacks of orthopnea and arrythmias. This is coincident with data published by Ahmed et al., 2008 who study the effect of recurrent hospitalizing of heart failure patients on the mortality, they found that, incident hospitalization due to worsening HF was associated with significant increase in all-cause and cardiovascular mortality in a wide spectrum of ambulatory patients with chronic mild to moderate systolic and diastolic HF [38].

These findings highlight the importance of HF hospitalization as a marker of disease progression and poor outcomes in HF, and emphasize on the need for prevention of HF hospitalization, and treatment strategies for hospitalized HF patients to improve post-discharge outcomes. These findings are important, as worsening HF is the number one reason for hospitalization for $\mathrm{HF}$ patients.

$\mathrm{HF}$ is a progressive disorder with poor prognosis. Common identifiable causes of HF hospitalizations include acute coronary syndrome, uncontrolled hypertension, arrhythmias and use of anti-arrhythmic drugs, pulmonary infections, and noncompliance with medications and diet. There is cumulative evidence that serum troponin levels may be elevated in $\mathrm{HF}$, which in turn may be associated with worsening HF, HF hospitalization, and mortality. Elevated serum troponin levels in acute HF have been associated with increased risk of subsequent mortality and hospitalizations. Other explanations for poor post-discharge outcomes include bed rest and restricted mobility during hospitalizations [39-41].

In the SOLVD trial, the survival benefit of Enalapril was observed only among the patients who were hospitalized at least once during the trial. Because treatment effects often depend on severity or stage of disease, a history of HF hospitalization may be used as inclusion criteria in future HF trials. This is important as event rates in contemporary systolic $\mathrm{HF}$ patients receiving optimal therapy and in those with diastolic HF (clinical HF with normal or near normal ejection fraction) are expected to be low. Future studies are needed to investigate whether 
cardiac resynchronization therapy during hospitalization and the prescription of beta-blockers at the time of hospital discharge might favorably reduce post-discharge mortality compared to patients without HF hospitalizations [42].

As a recent post-ho analysis of CHARM database demonstrated that post base-line "discharge for first hospitalization for HF" was independently associated with increased mortality [43].

In a paradigm of using a simple clinical questionnaire in our study to identify the risk among patients of HF, a large study made by Mikhail et al., in 2007 confirmed the risk between deterioration in clinical symptoms of HF-patients and mortality in the expanded project of "Health service and outcome research" using the famous Kansas City cardiomyopathy questionnaire through follow up 1358 patients of chronic HF and defined a definite relation between recurrent hospitalization, frequent orthopnea and lower limb swelling on reduction of life span and increased mortality [44].

\section{Limitations of the Study}

Like any non-randomized study, propensity score analysis cannot account for confounding due to unmeasured covariates. However our sensitivity analysis suggests that, our findings were rather insensitive to hidden biases. We are able to find near exact-matching for most of patients with HF-hospitalization. Also, in spite therapy for systolic HF has evolved since the DIG trial was conducted, we did not clarify the effect of therapy on HF-outcome and mortality and we had no data on use of beta-blockers, diuretic dosages and aldosterone antagonists is another limitation in our study. Indeed, the main crucial point in our study is relatively small number of cases and a shortterm follow up. Moreover, we did not directly estimate the exact number and percentage of mortality in our patients.

\section{Acknowledgements}

To the team work colleagues in Non-invasive unit of Cardiology Dept. El Minia University Hospital and the secretary employees of the department for easy call back and archiving process.

\section{REFERENCES}

[1] I. B. Goldstein and D. Shapiro, "The Cardiovascular Response to Postural Change as a Function of Race," Biological Psychology, Vol. 39, No. 2-3, 1995, pp. 173-186.

[2] C. J. Nardo, L. E. Chambless, K. C. Light, et al., "Descriptive Epidemiology of Blood Pressure Response to Change in Body Position: The ARIC Study," Hypertension, Vol. 33, No. 5, 1999, pp. 1123-1129.

doi:10.1161/01.HYP.33.5.1123
[3] The Consensus Committee of the American Autonomic Society and the American Academy of Neurology, "Consensus Statement on the Definition of Orthostatic Hypotension, Pure Autonomic Failure, and Multiple System Atrophy," Neurology, Vol. 46, No. 5, 1996 p. 1470. doi:10.1212/WNL.46.5.1470

[4] K. M. Rose, I. Holme, K. C. Light, et al., "Association between the Blood Pressure Response to a Change in Posture and the 6-Year Incidence of Hypertension: Prospective Findings from the ARIC Study," Journal of Human Hypertension, Vol. 16, No. 11, 2002, pp. 771-777. doi:10.1038/sj.jhh.1001482

[5] M. L. Eigenbrodt, K. M. Rose, D. J. Couper, et al., "Orthostatic Hypotension as a Risk Factor for Stroke: The Atherosclerosis Risk in Communities (ARIC) Study," Stroke, Vol. 31, 2000, pp. 2307-2313.

doi:10.1161/01.STR.31.10.2307

[6] H. Luukinen, K. Koski and P. Laippala, "Orthostatic Hypotension and the Risk of Myocardial Infarction in the Home-Dwelling Elderly," Journal of Internal Medicine, Vol. 255, No. 4, 2004, pp. 486-493. doi:10.1111/j.1365-2796.2004.01313.x

[7] K. M. Rose, I. Holme, K. C. Light, et al., "Association between the Blood Pressure Response to a Change in Posture and the 6-Year Incidence of Hypertension: Prospective Findings from the ARIC Study," Journal of Human Hypertension, Vol. 16, No. 11, 2002, pp. 771-777. doi:10.1038/sj.jhh.1001482

[8] M. L. Eigenbrodt, K. M. Rose, D. J. Couper, et al., "Orthostatic Hypotension as a Risk Factor for Stroke: The Atherosclerosis Risk in Communities (ARIC) Study, 19871996," Stroke, Vol. 31, 2000, pp. 2307-2313.

doi:10.1161/01.STR.31.10.2307

[9] H. Luukinen and K. Airaksinen, "Orthostatic Hypotension Predicts Vascular Death in Older Diabetic Patients," Diabetes Research and Clinical Practice, Vol. 67, No. 2, 2005, pp. 163-166. doi:10.1016/i.diabres.2004.06.009

[10] J. J. Smith, C. M. Porth and M. Erickson, "Hemodynamic Response to the Upright Posture," The Journal of Clinical Pharmacology, Vol. 34, No. 5, 1994, pp. 375-386.

[11] C. J. Mathias and J. R. Kimber, "Postural Hypotension: Causes, Clinical Features, Investigation, and Management," Annual Review of Medicine, Vol. 50, 1999, pp. 317-336. doi:10.1146/annurev.med.50.1.317

[12] L. M. Allcock, R. A. Kenny, U. P. Mosimann, et al., “Orthostatic Hypotension in Parkinson's Disease: Association with Cognitive Decline," International Journal of Geriatric Psychiatry, Vol. 21, No. 8, 2006, pp. 778-783. doi:10.1002/gps.1562

[13] S. Gates, J. D. Fisher, M. W. Cooke, et al., "Multifactorial Assessment and Targeted Intervention for Preventing Falls and Injuries among Older People in Community and Emergency Care Settings: Systematic Review and MetaAnalysis,” British Medical Journal, Vol. 336, No. 19, 2008, pp. 130-133.

[14] M. A. Weber, N. K. Wenger and S. Scheidt, "A Focus on Heart Failure and Other Age-Related Cardiovascular Conditions," American Journal of Geriatric Cardiology, Vol. 14, No. 5, 2005, pp. 221-223. 


\section{doi:10.1111/j.1076-7460.2005.04223.x}

[15] C. L. Avery, P. P. Chang, W. D. Rosamond, et al., "The Population Burden of Heart Failure Attributable to Modifiable Risk Factors: The ARIC (Atherosclerosis Risk in Communities) Study," American College of Cardiology, Vol. 60, No. 17, 2012, pp. 1640-1646. doi:10.1016/j.jacc.2012.07.022

[16] K. M. Rose, H. A. Tyroler, C. J. Nardo, et al., "Orthostatic Hypotension and the Incidence of Coronary Heart Disease: The Atherosclerosis Risk in Communities Study," American Journal of Hypertension, Vol. 13, No. 6, 2000, pp. 571-578. doi:10.1016/S0895-7061(99)00257-5

[17] K. M. Rose, M. L. Eigenbrodt, R. L. Biga, et al., "Orthostatic Hypotension Predicts Mortality in Middle-Aged Adults: The Atherosclerosis Risk in Communities (ARIC) Study," Circulation, Vol. 114, No. 7, 2006, pp. 630-636. doi:10.1161/CIRCULATIONAHA.105.598722

[18] M. L. Eigenbrodt, K. M. Rose, D. J. Couper, et al., "Orthostatic Hypotension as a Risk Factor For Stroke: The Atherosclerosis Risk in Communities (ARIC) Study, 1987-1996," Stroke, Vol. 31, 2000, pp. 2307-2313. doi:10.1161/01.STR.31.10.2307

[19] A. I. Vinik, B. Aysin and J. Colombo, "Dynamic Enhanced Frequency Domain Analysis Indicates a Significant Decline in Autonomic Function before Age 50," Diabetes Technology Conference, San Francisco, 2005.

[20] A. I. Vinik, R. Freeman and T. Erbas, "Diabetic Autonomic Neuropathy," Seminars in Neurology, Vol. 23, No. 4, 2003, pp. 365-372. doi:10.1055/s-2004-817720

[21] S. M. Grundy, I. J. Benjamin, G. L. Burke, et al., "Diabetes and Cardiovascular Disease: A Statement for Healthcare Professionals from the American Heart Association," Circulation, Vol. 100, No. 13, 1999, pp. 1134-1146. doi:10.1161/01.CIR.100.10.1134

[22] R. Freeman, "Treatment of Orthostatic Hypotension," Seminars in Neurology, Vol. 23, No. 4, 2003, pp. 435-442. doi:10.1055/s-2004-817727

[23] B. Aysin, E. Aysin and A. Vinik, "Diabetes May Accelerate the Onset of Orthostasis," The 6th Annual Diabetes Technology Meeting, Atlanta, 2006.

[24] R. R. Arora, E. Aysin and J. Colombo, "Therapeutic Implications of Sympathetic Stimulus in Orthostatic Patients: Measured by Spectral Domain Analysis," AHA Scientific Sessions, Orlando, 2007.

[25] C. Borst, W. Weiling, J. F. van Brederode, A. J. Dunning, et al., "Mechanisms of Initial Heart Rate Response to Postural Change," American Journal of Physiology, Vol. 243, No. 5, 1982, pp. 676-681.

[26] S. Maule, E. Catalfamo, S. D. Colle, et al., "Cardiovascular Autonomic Function in 422 Patients with Orthostatic Symptoms," American Journal of Hypertension, Vol. 16, 2003, p. 505.

[27] American Academy of Neurology, "Clinical Autonomic Testing Report of the Therapeutics and Technology Assessment Subcommittee of the American Academy of Neurology, Neurology, Vol. 46, No. 3, 1996, pp. 873-880.

[28] A. M. Aring, D. E. Jones and J. M. Falko, "Evaluation and Prevention of Diabetic Neuropathy," American Family
Physician, Vol. 71, No. 11, 2005, pp. 2123-2130.

[29] J. J. Smith, C. M. Porth and M. Erickson, "Hemodynamic Response to the Upright Posture," The Journal of Clinical Pharmacology, Vol. 34, No. 5, 1994, pp. 375-386.

[30] W. B. Applegate, B. R. Davis, H. R. Black, et al., "Prevalence of Postural Hypotension at Baseline in the Systolic Hypertension in the Elderly Program (SHEP) Cohort," Journal of the American Geriatrics Society, Vol. 39, No. 11, 1991, pp. 1057-1064.

[31] H. Luukinen, K. Koski and P. Laippala, "Orthostatic Hypotension and the Risk of Myocardial Infarction in the Home-Dwelling Elderly," Journal of Internal Medicine, Vol. 255, No. 4, 2004, pp. 486-493. doi:10.1111/j.1365-2796.2004.01313.x

[32] C. A. Ray and K. D. Monahan, "Aging Attenuates the Vestibulosympathetic Reflex in Humans," Circulation, Vol. 105, No. 8, 2002, pp. 956-961. doi: $10.1161 / \mathrm{hc} 0802.104289$

[33] C. Shin, R. D. Abbott, H. Lee, et al., "Prevalence and Correlates of Orthostatic Hypotension in Middle-Aged Men and Women in Korea: The Korean Health and Genome Study," Journal of Human Hypertension, Vol. 18, 2004, pp. 717-723. doi:10.1038/sj.jhh.1001732

[34] V. Litvinov, I. Alon, et al., "Seating-Induced Postural Hypotension Is Common in Older Patients with Decompensated Heart Failure and May Be Prevented by Lower Limb Compression Bandaging," Circulation, Vol. 55, No. 2, 2009, pp. 138-144.

[35] A. Fedorowski, B. Hedblad and O. Melander, "Directionality of Blood Pressure Response to Standing May Determine Development of Heart Failure: Prospective Cohort Study," European Journal of Heart Failure, Vol. 13, No. 5, 2011, 496-503. doi:10.1093/eurihf/hfr013

[36] A. Fedorowski, G. Engström and B. Hedblad, "Orthostatic Hypotension Predicts Incidence of Heart Failure: The Malmö Preventive Project," American Journal of Hypertension, Vol. 23, No. 11, 2010, pp. 1209-1215. doi:10.1038/ajh.2010.150

[37] K. Potocka-Plazak and W. Plazak, "Orthostatic Hypotension in Elderly Women with Congestive Heart Failure," Aging (Milano), Vol. 13, No. 5, 2001, pp. 378-84.

[38] M. D. Ali-Ahmed, R. M. Allman, C. Gregg, et al., "Incident Heart Failure Hospitalization and Subsequent Mortality in Chronic Heart Failure: A Propensity-Matched Study," Journal of Cardiac Failure, Vol. 14, No. 3, 2008 , pp. 211-218. doi:10.1016/j.cardfail.2007.12.001

[39] M. Gheorghiade, F. Zannad, G. Sopko, et al., "Acute Heart Failure Syndromes: Current State and Framework for Future Research," Circulation, Vol. 112, No. 65, 2005, pp. 3958-3968. doi:10.1161/CIRCULATIONAHA.105.590091

[40] G. D. Schiff, S. Fung, T. Speroff, et al., "Decompensated Heart Failure: Symptoms, Patterns of Onset, and Contributing Factors," American Journal of Medicine, Vol. 114, No. 8, 2003, pp. 625-630. doi:10.1016/S0002-9343(03)00132-3

[41] E. R. Perna, S. M. Macin, J. P. Cimbaro-Canella, et al., "Minor Myocardial Damage Detected by Troponin T Is a 
Powerful Predictor of Long-Term Prognosis in Patients with Acute Decompensated Heart Failure," International Journal of Cardiology, Vol. 99, No. 2, 2005, pp. 253-261. doi:10.1016/j.ijcard.2004.01.017

[42] The SOLVD Investigators, "Effect of Enalapril on Survival in Patients with Reduced Left Ventricular Ejection Fractions and Congestive Heart Failure," The New England Journal of Medicine, Vol. 325, 1991, pp. 293-302. doi:10.1056/NEJM199108013250501

[43] P. M. Rothwell, "Treating Individuals 2. Subgroup Ana- lysis in Randomised Controlled Trials: Importance, Indications, and Interpretation," Lancet, Vol. 365, No. 9454, 2005, pp. 176-186. doi:10.1016/S0140-6736(05)17709-5

[44] M. Kosiborod, G. E. Soto, P. G. Jones, et al., "Outcomes Research, Identifying Heart Failure Patients at High Risk for Near-Term Cardiovascular Events With Serial Health Status Assessments," Circulation, Vol. 115, No. 15, 2007, pp. 1975-1981. 